\title{
IV. Alutizen.
}

\section{Gemeinfamer Etat für hoch= und Zutederwald imnerhalb eines Wirt: fhaftsganzen.}

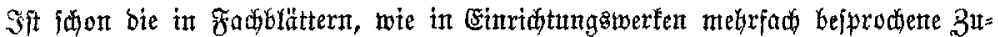
fammenfafiung bes (stat ber Scauptnubungen mit bem Etat ber Bwifdennutüungen für

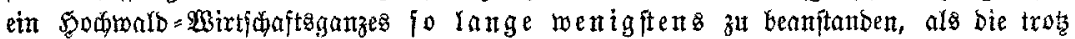

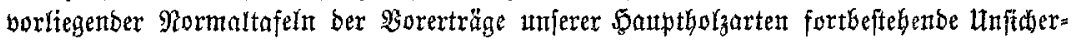

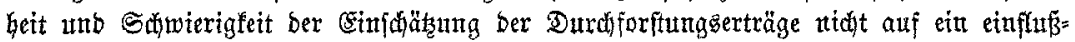

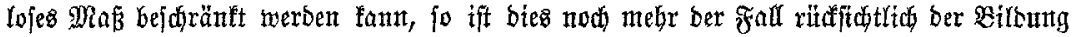

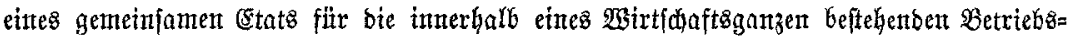

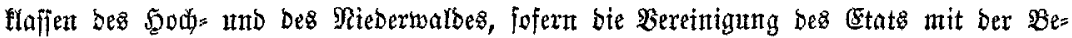

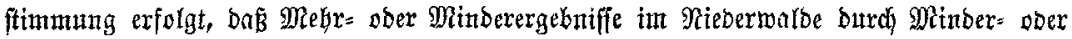

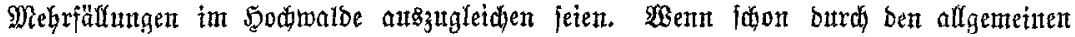

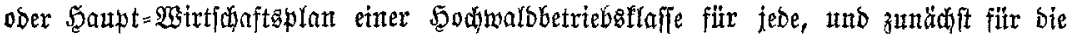

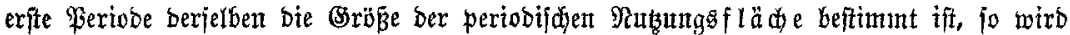

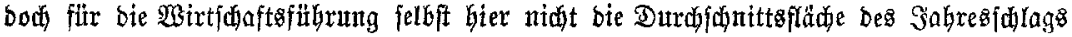
in erfter Rinie mażgebend; ber berednete jührlide Materialetat iftes, welder zu=

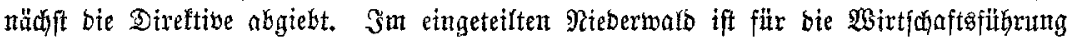

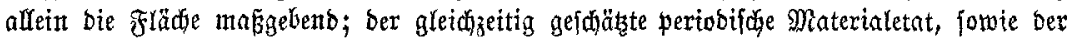
bieran abgelettete Sahresetat an Maffe, haben für fite leine Bebeutung. Fhir bie Bubget

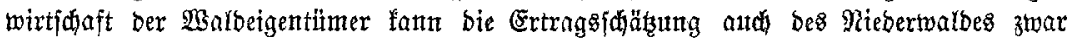

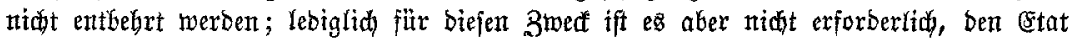

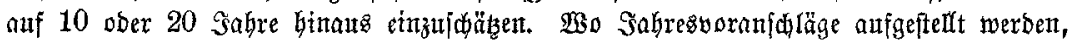

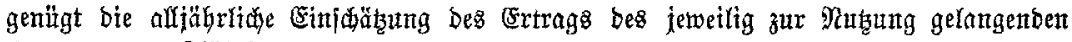

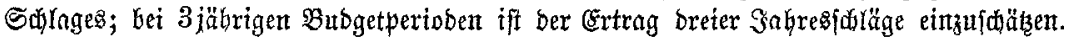

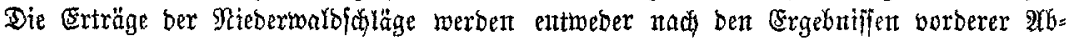

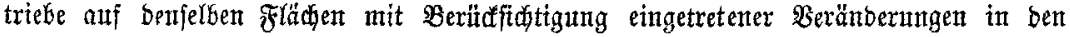

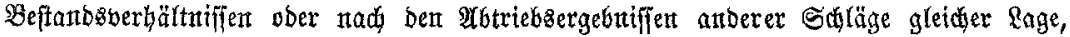

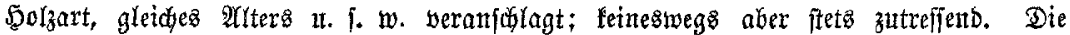
Sctätzungefehler bewegen fitht oft in febr weiten (5rentzen. Soldien Feglern, welche

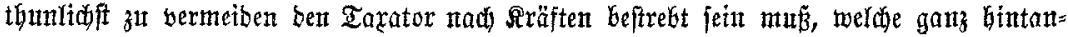

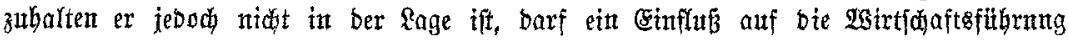

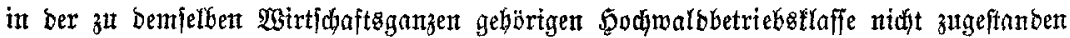

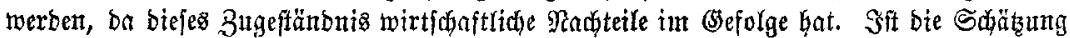
Des Rieberwalbertragg zu bod, bleibt aljo bas Ergebnis binter ber Sdäbung zurilid, io

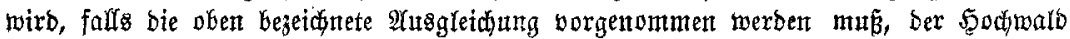

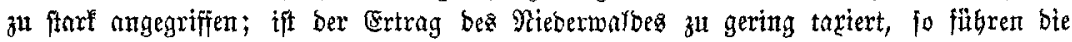

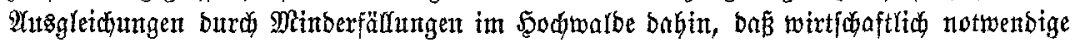

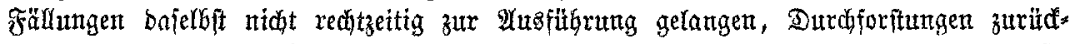

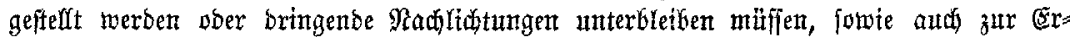

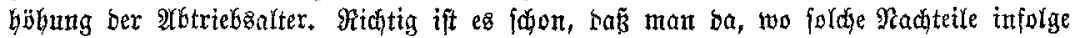

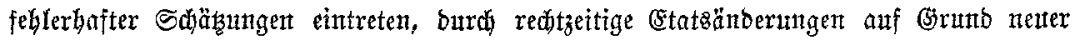

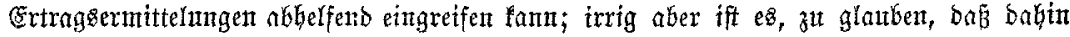

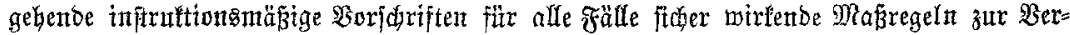

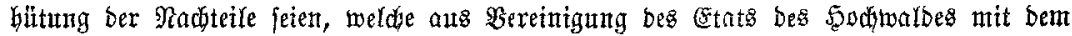

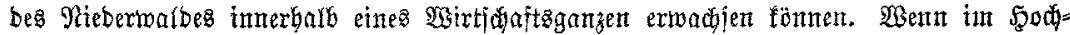




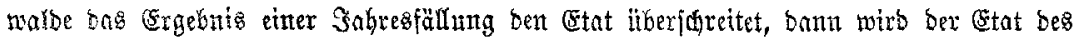

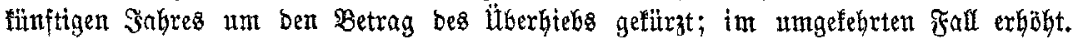

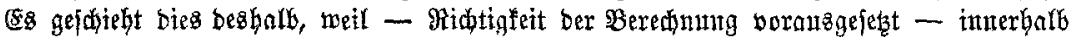

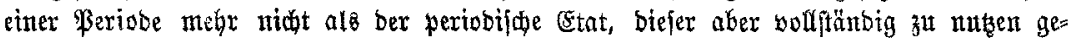
ffattet if̣t. War sie Beredinung bes Etat riditig, fo ftimmen auth bejitglid bes

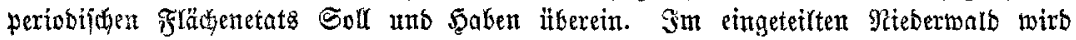
alljälyrlids ein Sajlng geerntet. Doñ bie Salüge fogentantte Proportiontalläläge unt

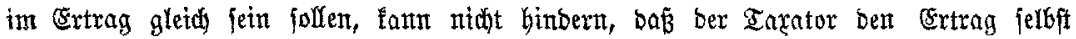
unridtig bemist. Şat man aber, jagt Cotta, in ber Befftumung bes Ertrngo geirrt,

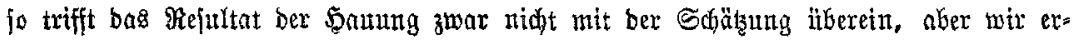

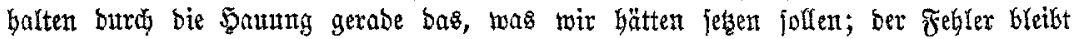

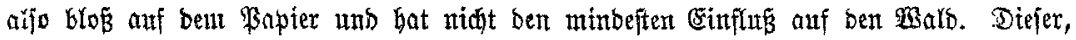

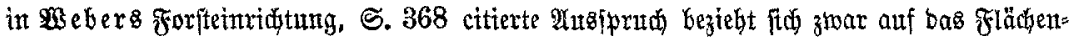

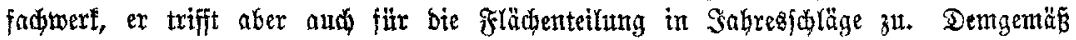

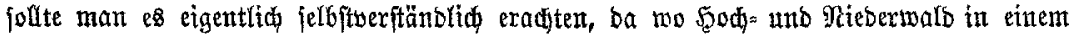

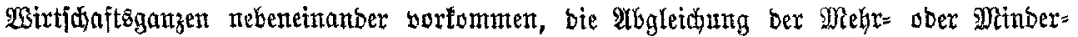
ergebniffe bes Niebertwalbes gegeniiber ber borliegenten Shäbung nidjt in Sadjwalbe,

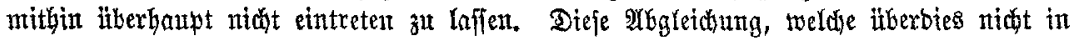

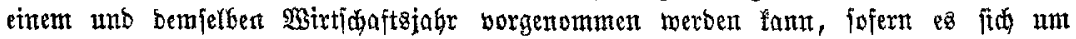

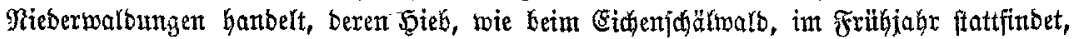
mobei bie Winterfällung im sodfwalte vorauggegangen ift, entGebrt alfo ber interen

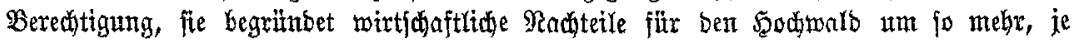

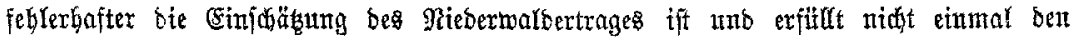

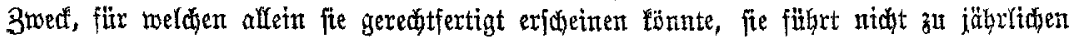
gleiden Erträgen bes wirtjonftżganzen, weil jebes Sabr eine neue Wbmeiding erfolgt.

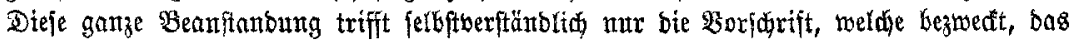

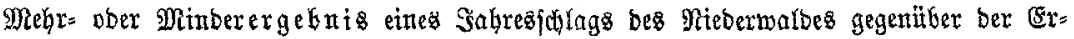

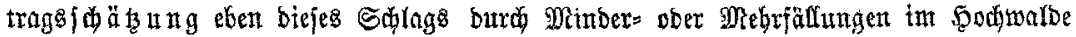

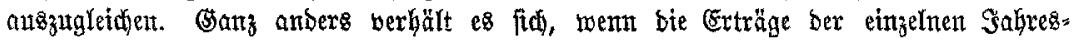

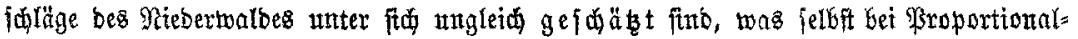
forlägen während bes exften Umtriebs, alfo bei now nidit vorliegender Biornalität ber

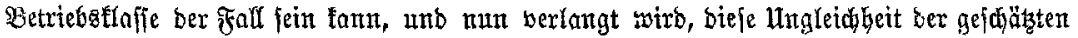

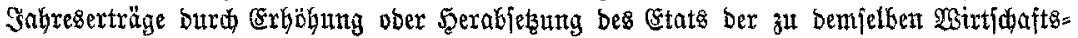

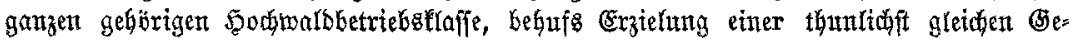

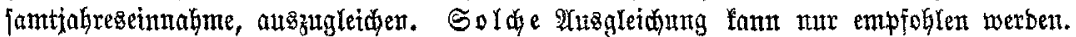

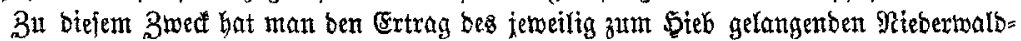

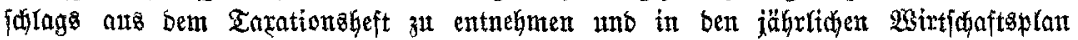

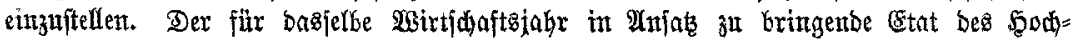
watbes befteht Dant in ber Differenz zwifdien bem angeworfenten einfleitidien Sabres.

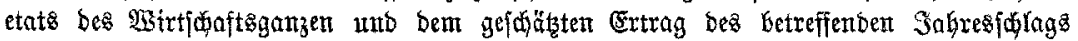

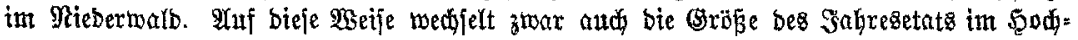
walbe, aber bie Summe aller fo bemeffenen Sabrezetatz Der Spobmalbbetriebstafife ent=

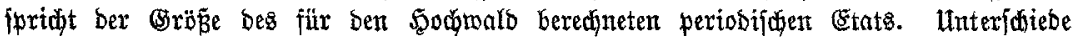

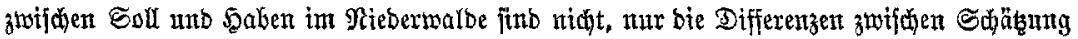

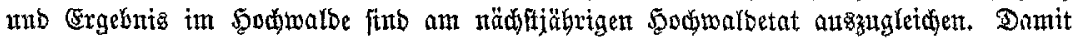

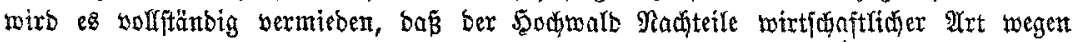

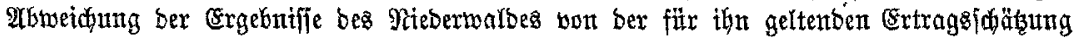

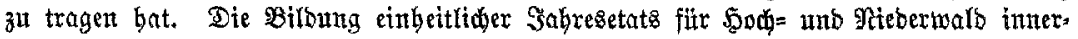




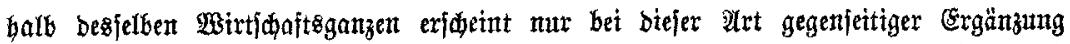
gerechtfertigt; andernfalls bält mon beibe Etats befier getrennt. Werben im Nieberwalb and Durdforftungen gefïhrt, fo wirb bamit an gegenwärtiger Sache nidt geänbert.

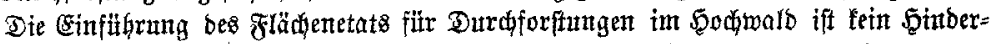
nis bafïr, in einzelnen Sahren bie Sautstnukungen auf Roften ber Durdforftungent

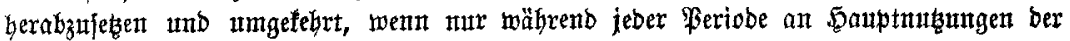

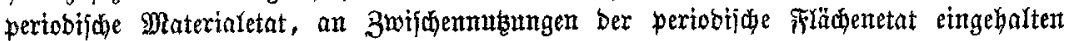

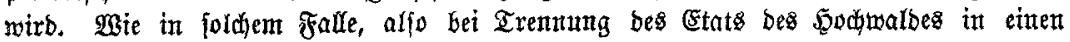
Material = und einen Flähjentat, bet Bilbung einheitlidjen bezas. gemeinfamen Sahregs

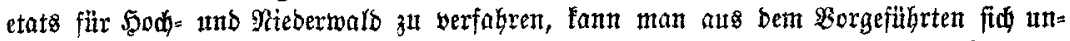
folmer lebbet ableiten.

S.

\section{Gillfstabellen für forfttaratoren.}

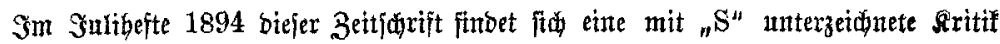

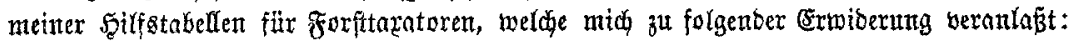

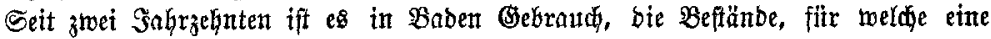

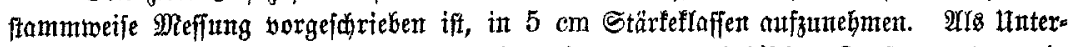

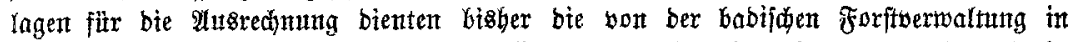

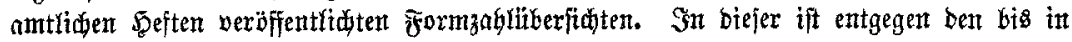

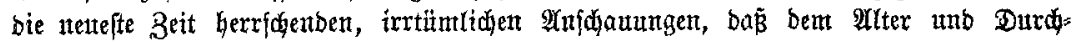
mefiex ein befonberer Einflup zufonme, bie Baumformzahl als nur von bex Shöbe ab= bängig bargefiellt.

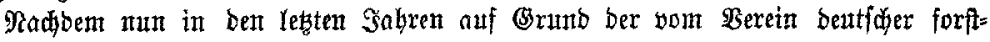

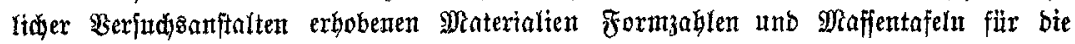

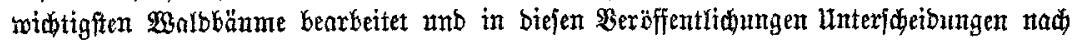
IItergflafien und $\mathfrak{B a d j o t u m g g e b i e t e n ~ g e m a d i t , ~ b i e ~ M o i j e n t a f e l n t ~ a b e r ~ i n ~} 1$ ober $2 \mathrm{~cm}$

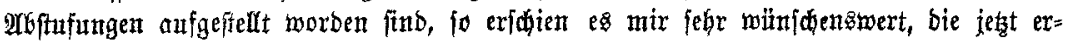
mittelten Formzablett unfern Selzmaffenberedinungen zu (sruntbe zut legen, zugleid) aber

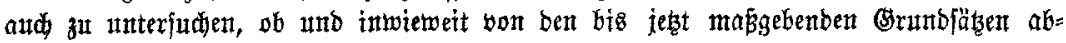
zutweident fei ober nicht.

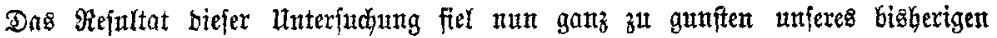

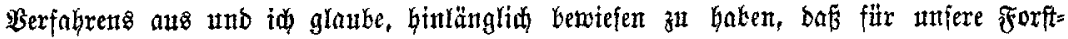
eintridutuggarfeiten bie Baumformzahl als nur von ber Säbe abbängig Getradtet

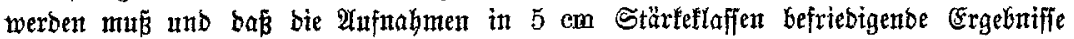
fiefern.

Şerr "S" bebauptet num: "Der Berfaffer greift bie neueren Maffentafeln uno ihre

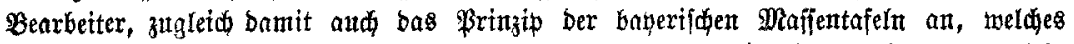
jene zum $\mathfrak{T}$ eif nidjt anfgeten wollten, braud)t aber bie (Ergebniffe ibrer mithjamen 2 rbeit, obne befferes bieten zat tönnen." - unb an einet andern Stefle:

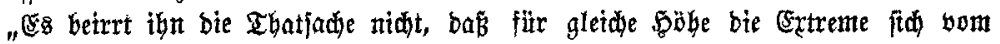
Durofjinitt oft $\mathrm{um} \pm 20$ fis 25 p@.t entfernen."

Die $\mathfrak{B} e$ hantptung, ba $\tilde{\beta}$ id bie Bearbeiter ber netteren Maffentafeln angreife, ift

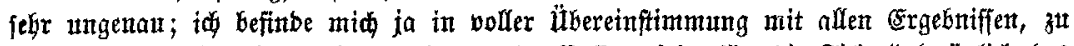

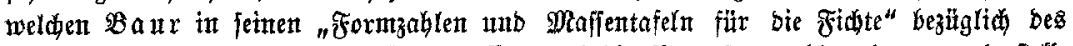

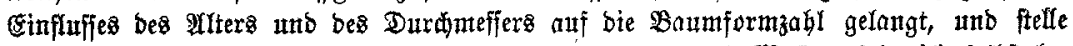

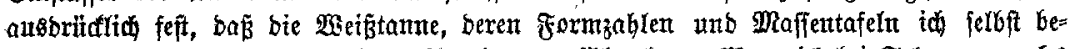

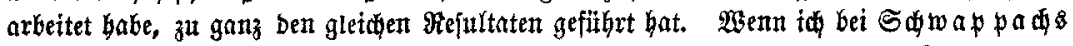

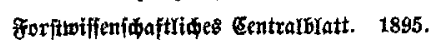

